Check for updates

Cite this: RSC Adv., 2017, 7, 40581

Received 30th June 2017 Accepted 30th July 2017

DOI: $10.1039 / \mathrm{c} 7 \mathrm{ra0} 7239 \mathrm{~b}$

rsc.li/rsc-advances

\section{Upgrading of palmitic acid over MOF catalysts in supercritical fluid of $\boldsymbol{n}$-hexane $\dagger$}

\author{
Xiao Fang, ${ }^{a}$ Yanchun Shi, ${ }^{a}$ Kejing Wu, ${ }^{a}$ Junmei Liang, ${ }^{a}$ Yulong Wu (D) *ab \\ and Mingde Yang ${ }^{a}$
}

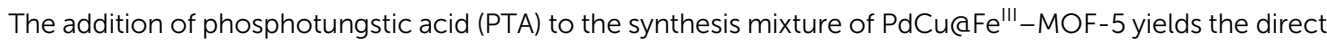

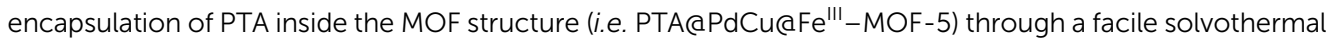
approach. The deoxygenation reaction of palmitic acid has been investigated over PdCu@Fe ${ }^{\prime \prime \prime}-M O F-5$ and PTA@PdCu@Fe ${ }^{\prime \prime \prime}-$ MOF-5 under a hydrogen atmosphere in the supercritical fluid (SCF) of $n$-hexane. The results showed that palmitic acid can be converted completely at $240{ }^{\circ} \mathrm{C}$ on PTA@PdCu@Fe ${ }^{\prime \prime \prime}-\mathrm{MOF}-5$ with a high selectivity of hexadecane. Owning to the improvement of acidity of the MOF catalyst by the encapsulation of PTA inside the hollow octahedral nanostructures of PdCu@Fe ${ }^{\text {III}}-\mathrm{MOF}-5$, the selectivity for hexadecane over the PTA@PdCuaFe III-MOF-5 catalyst is higher than that over PdCuaFe'II-MOF-5. The excellent performance in the catalytic hydrodeoxygenation (HDO) of palmitic acid is associated with the synergistic effect between yolk-shell PTA@PdCu@Fe III-MOF-5 nanostructures and SCF medium.

\section{Introduction}

Concerns about the environment and rising costs of fossil fuels have stimulated the search for renewable supplements to fossil fuels. Biomass and its derivatives can provide the sustainable production of liquid fuels and valuable chemicals. ${ }^{\mathbf{1 - 4}}$ Algae is mainly composed of proteins, lipids and polysaccharides. Only lipids in algae can be used by traditional extraction esterification methods for the preparation of biodiesel, ${ }^{1}$ while thermochemical conversion technology can realize the usage of all components of algae. The resulting liquid product can be called bio-oil. ${ }^{2}$ Algae-derived biofuel is regarded as the only current renewable source of oil that could meet the global demand for transport fuels and high added valued chemicals. ${ }^{3,4}$ However, large amounts of oxygen-containing organic compounds exist in the algae-based bio-oil and lead to polymerization, low calorific value, poor stability and strong corrosion..$^{4,5}$ As a result, the bio-oil cannot be directly used as transportation fuels without upgrading C16-C18 long-chain fatty acids (the main components exist in algal bio-oil). ${ }^{6,7}$ Catalytic cracking, catalytic esterification and HDO are the main methods for upgrading bio-oil. In particular, the HDO method, which can fundamentally overcome the disadvantage of high oxygen content in biooil, has become the focus of research recently. However, some shortages still exist in the HDO of algal-based bio-oil. The

${ }^{a}$ Institute of Nuclear and New Energy Technology, Tsinghua University, Beijing 100084, PR China. E-mail: wylong@tsinghua.edu.cn; Fax: +86 10-69771464; Tel: +86 01089796163

${ }^{b}$ Beijing Engineering Research Center for Biofuels, Beijing 100084, China

$\uparrow$ Electronic supplementary information (ESI) available. See DOI: $10.1039 / \mathrm{c} 7 \mathrm{ra07239b}$ catalyst efficiency is relatively low as the large molecules like C16-C18 long-chain fatty acids are hard to spread to the active sites in the pore of the catalyst in conventional medium. In spite of low hydrogen solubility in traditional reaction medium, the high temperature needed in hydrogenation reactions further reduces the hydrogen solubility. Yet the hydrogen dissolvability remains limited even under 13-18 $\mathrm{MPa}$. The high hydrogen pressure restricted the gas-liquid mass transfer process as well as decreasing the safety of the reaction. The aggregation of reactants due to slow diffusion caused carbon deposit on the activity sites leading to the deactivation of catalyst.

The key parameters affect the activity and product selectivity in porous catalysts are adsorption/desorption and pore-transport. ${ }^{8,9}$ Due to the unique tunable properties (e.g. diffusivity, transport ability), SCFs have with a broad range of applications and dramatically out-performed traditional solvents. ${ }^{10-13}$ SCFs had been utilized as reaction medium for the hydrogenation upgrading of model compounds recently. ${ }^{\mathbf{1 4 - 1 8}}$ Supercritical methanol technology has proved to be effective for the upgrading of low-boiling fraction of bio-oil. ${ }^{14}$ The upgrading of crude bio-oil was conducted in supercritical ethanol by Dang et al. ${ }^{15}$ Hydroprocessing of model bio-oil compound $p$-cresol in both water and supercritical $n$-heptane indicated that the reaction were mass-transfer controlled in water while in supercritical $n$-heptane the mass-transfer limitations were eliminated due to the complete $\mathrm{H}_{2}$ miscibility and enhanced pore diffusivities. ${ }^{\mathbf{1 6}}$ Deoxygenation of the model bio-oil compound nonanal over $\mathrm{Pt} / \mathrm{Al}_{2} \mathrm{O}_{3}$ in supercritical $n$-hexane at $300{ }^{\circ} \mathrm{C}$ and a pressure range of $0.7-8 \mathrm{MPa}$ showed a high solubility of $\mathrm{H}_{2}$, and obtained $60 \%$ conversion and $40 \%$ selectivity to C8 and C9 hydrocarbons. ${ }^{17}$ The upgrading of bio-oil prepared 
from hydrothermal liquefaction of cornstalks over $\mathrm{Ni} / \mathrm{ZrO}_{2}$ in supercritical cyclohexane under $573 \mathrm{~K}$ and $5 \mathrm{MPa} \mathrm{H}_{2}$ obtained $81.6 \%$ yield of hydrocarbons with an excellent quality. ${ }^{18}$ The studies above are based on the model compounds in bio-oil comes from terrestrial biomass. The catalytic HDO of model compounds in algal-based bio-oil like palmitic acid in SCF media has not yet been reported. The properties of SCFs enable a good compatibility of the reactants hydrogen and macromolecules like C16 long-chain fatty acids in SCF and more likely to form a single phase. The pore-transport of the reactants to the active sites of catalyst was promoted. Meanwhile, the products can be removed timely, thus alleviating the gas-liquid mass transfer limit existed in HDO reaction. Avoiding highpressure hydrogen the security was also improved. ${ }^{9}$

MOFs as a unique class of porous materials are widely applied to catalysis, separation, gas storage and molecular recognition ${ }^{\mathbf{1 9 , 2 0}}$ and are typically characterized by large surface areas, tunable and uniform pore structures, and interconnected three-dimensional cavities accessible through small pore windows. ${ }^{21-23}$ These characteristics enable MOFs to confine nanoparticles (NPs) e.g. polyoxometalates (POMs) and metal nanoparticles (MNPs) like $\mathrm{Au}, \mathrm{Pd}, \mathrm{Pt}$ and $\mathrm{Cu}$ etc. ${ }^{21}$ Interaction among the incorporated MNPs were impeded, thus avoiding agglomeration and detachment of the MNPs, whereas the smooth diffusion of reactants into and out of MOFs were guaranteed. ${ }^{21}$ The hollow cage of MOFs could facilitate the diffusion of substrates onto the internal metal surface as well as the desorption of products. ${ }^{2,25}$ Recently, MOFs used for catalytic hydrogenation reaction had been systematically explored. ${ }^{26-31}$ Zhao et al. showed that MOFs with special sandwich nanostructures had an excellent catalytic performance for the hydrogenation of $\alpha, \beta$-unsaturated aldehydes. ${ }^{28}$ Pd@MOF-5 were proved to be a moderately active catalyst for the hydrogenation of cyclooctene. ${ }^{27}$ The hydrogenation of 2,3,5-trimethylbenzoquinone using $\mathrm{Pd}_{28}$ @UiO-66$\mathrm{NH}_{2}$ has been investigated in the literature. ${ }^{29}$ The ultrasmall Pd NPs encaged in amine-functionalized UiO-66 in HDO of vanillin in water indicated that $100 \%$ conversion of vanillin with exclusive selectivity for 2-methoxy-4-methylphenol were achieved under mild conditions. ${ }^{30}$ The heterometallic MOF systems bearing two metals e.g. the yolk-shell PdCu@Fe ${ }^{\mathrm{III}}$ MOF-5 nanostructures were successfully designed though a facile solvothermal method and were found to show highly improved catalytic performance in the liquid-phase selective hydrogenation of 1-chloro-2-nitrobenzene relative to the pure yolk. ${ }^{31,32}$ In short, MOFs encapsulated with MNPs had displayed remarkable catalytic hydrogenation performance due to the unique porous structure and hydrogen storage capacity. POMs consisted of heteropolyanions are complex Brønsted acids and can be encapsulated within the pores of MOF. ${ }^{33}$ Studies of many POMs based MOFs have been reported. ${ }^{34}$ Incorporating the acid functionality of PTA in MIL-101 improved the acidity of MOFs, ${ }^{35,36}$ thereby contributing to the dehydration of carbohydrates. ${ }^{37}$ Encapsulating MOFs with POMs makes them excellent candidates as heterogeneous catalysts. $^{38}$ The hydrogenation of long-chain fatty acid by MOFs has been rarely reported.
Exploiting SCF as the reaction medium and MOFs with both microporous and mesoporous structures as the catalyst are supposed to conquer the above existed deficiencies about catalytic HDO of algal-based bio-oil. In this study, palmitic acid is selected as the model compound of algal-based bio-oil. The encapsulation of PTA to the synthesis mixture of PdCu@Fe ${ }^{\text {III }}$ MOF-5 obtained PTA@PdCu@Fe ${ }^{\mathrm{III}}-\mathrm{MOF}-5$ through one-pot solvothermal approach. ${ }^{24}$ The as-prepared yolk-shell PTA@Pd$\mathrm{Cu} @ \mathrm{Fe}^{\mathrm{III}}-\mathrm{MOF}-5$ are used for the catalytic HDO reaction of palmitic acid with the SCF of $n$-hexane $\left(T_{\mathrm{c}}=234.5{ }^{\circ} \mathrm{C}, P_{\mathrm{c}}=\right.$ $3 \mathrm{MPa}$ ) as reaction medium. The remarkable performance of the catalytic HDO reactions attributes to the excellent solubility and diffusibility of SCF media, the high hydrogen storage capacity and special pore structure of the MOF catalysts. ${ }^{39-41}$

\section{Experimental}

\subsection{Materials}

Palmitic acid (99\%), hexadecanol ( $\geq 99.5 \%)$, hexadecanal $(\geq 99.5 \%)$, hexadecane ( $\geq 99.5 \%)$, pentadecane ( $\geq 99.5 \%), N, N$ dimethylformamide (DMF) ( $\geq 99.5 \%)$, ethanol $(\geq 99.5 \%)$, $\mathrm{Pd}(\mathrm{acac})_{2}, \quad \mathrm{Cu}(\mathrm{acac})_{2}, \quad \mathrm{Fe}(\mathrm{acac})_{3}, \quad n$-hexane and PTA were purchased from Aladdin Chemistry Co., Ltd. (Shanghai, China). $\mathrm{Zn}\left(\mathrm{NO}_{3}\right)_{2} \cdot 6 \mathrm{H}_{2} \mathrm{O}, \mathrm{H}_{2} \mathrm{BDC}$ and PVP $\left(M_{\mathrm{w}}=30000\right)$ were bought from Shanghai Chemical Reagents Co., Ltd. (Shanghai, China). All reagents were used as-received without further purification.

\subsection{Synthesis of catalysts}

2.2.1 PTA@Fe ${ }^{\mathrm{III}}-\mathrm{MOF}-5$ hollow octahedral nanostructures. $\mathrm{Fe}^{\mathrm{III}}$-MOF-5 encapsulating PTA (donated as PTA@Fe ${ }^{\mathrm{III}}-\mathrm{MOF}-5$ ) was synthesized using a facile solvothermal method. ${ }^{32}$ Typically, $60 \mathrm{mg} \mathrm{Fe}(\text { acac })_{3}, 46.4 \mathrm{mg} \mathrm{Zn}\left(\mathrm{NO}_{3}\right)_{2} \cdot 6 \mathrm{H}_{2} \mathrm{O}$, $9.6 \mathrm{mg}$ $\mathrm{H}_{2} \mathrm{BDC}$, $9.6 \mathrm{mg}$ PTA and $200 \mathrm{mg}$ PVP were dissolved in $25.6 \mathrm{~mL}$ DMF-ethanol mixture $(\mathrm{v} / \mathrm{v}=5: 3)$ under magnetic stirring for $10 \mathrm{~min}$ at room temperature. The resulting homogeneous solution was transferred to a $40 \mathrm{~mL}$ Teflon-lined stainless-steel autoclave. After heating the sealed vessel at $100{ }^{\circ} \mathrm{C}$ for $6 \mathrm{~h}$ it was cooled to room temperature. Separation and purification of the products via centrifugation was the same as the treating of PTA@MOF-5.

\subsubsection{PTA@PdCu@Fe $\mathrm{III}_{\text {III }}$ MOF-5 hollow nanostructures.} Briefly, a $2 \mathrm{~mL}$ nanoparticles solution of desired concentration and $200 \mathrm{mg}$ PVP were mixed with $23.6 \mathrm{~mL}$ DMF-ethanol mixture $(\mathrm{v} / \mathrm{v}=5: 3)$ under magnetic stirring for 10 minutes at room temperature. Then, $60 \mathrm{mg} \mathrm{Fe}(\text { acac })_{3}, 46.4 \mathrm{mg} \mathrm{Zn}\left(\mathrm{NO}_{3}\right)_{2} \cdot 6 \mathrm{H}_{2} \mathrm{O}$, $9.6 \mathrm{mg} \mathrm{H} \mathrm{H}_{2} \mathrm{BDC}$ and $9.6 \mathrm{mg}$ PTA were put into the above solution under magnetic stirring for another $10 \mathrm{~min}$. The resulting solution was transferred to a $40 \mathrm{~mL}$ Teflon-lined stainless-steel autoclave. The sealed vessel was then heated at $100{ }^{\circ} \mathrm{C}$ for $6 \mathrm{~h}$ before it was cooled to room temperature. Separation and purification of the products was the same as described above.

MOF-5 (Fig. S1 of the ESI data†), PTA@MOF-5 (Fig. S2 of the ESI data $\dagger$ ), Fe ${ }^{\mathrm{III}}-\mathrm{MOF}-5$ (Fig. S3 of the ESI data $\dagger$ ), $\mathrm{PdCu@Fe} \mathrm{FI}^{\mathrm{III}}$ MOF-5 (Fig. S4 of the ESI data†), Pd NPs@Fe ${ }^{\mathrm{III}}-\mathrm{MOF}-5$ (Fig. S5 of the ESI data $\dagger$ ), Au NPs@Fe ${ }^{\mathrm{III}}-\mathrm{MOF}-5$ (Fig. S6 of the ESI data $\dagger$ ), PdCu dendrites (Fig. S7 of the ESI data $\dagger$ ), Pd NPs (Fig. S8 of the 
ESI data $\dagger$ ) and Au NPs (Fig. S9 and S12 of the ESI data $\dagger$ ) were synthesized according the previous reports. ${ }^{32,42}$

\subsection{Experiments and analysis of the products}

The catalytic hydrogenation experiments of palmitic acid were conducted in a $100 \mathrm{~mL}$ batch autoclave heated with an external electrical furnace. And the temperature was measured by a thermocouple and controlled at $\pm 1{ }^{\circ} \mathrm{C}$. In a typical experiment, palmitic acid $(0.3 \mathrm{~g})$ dissolved in $n$-hexane $(30 \mathrm{~mL})$ and the catalyst $(0.1 \mathrm{~g})$ were added into the reactor, before pressurizing with appropriate amount of $\mathrm{H}_{2}$ the autoclave was purged with $\mathrm{H}_{2}(0.2 \mathrm{MPa})$ for three times to remove air at ambient temperature. The products were confirmed by GC-MS (Agilent $6820 \mathrm{~N}$ series) and quantitatively analyzed by the gas chromatograph (GC) equipped with a GsBP-Inowax $(30 \mathrm{~m} \times$ $0.32 \mathrm{~mm}, 0.25$ Micron) column and flame ionization detector (FID) with $\mathrm{He}\left(10 \mathrm{~mL} \mathrm{~min}^{-1}\right)$ as carrier gas and injector temperature $320{ }^{\circ} \mathrm{C}$.

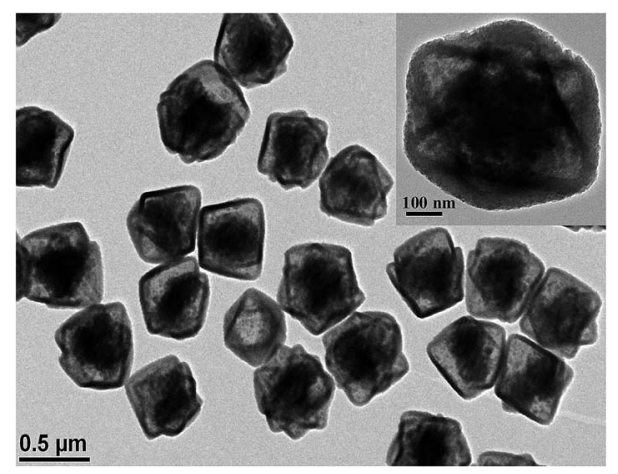

Fig. 1 TEM image of PTA@Fe ${ }^{\prime \prime \prime}-M O F-5$.

\section{Results and discussion}

\subsection{Catalysts characterization}

The morphology and structural features of as-prepared PTA@Fe $\mathrm{III}^{\mathrm{III}} \mathrm{MOF}-5$ and PTA@PdCu@Fe ${ }^{\mathrm{III}}-\mathrm{MOF}-5$ hollow nanospheres were characterized by transmission electron microscopy (TEM). As exhibited in Fig. S2, $\dagger$ PTA@MOF-5 remains a roughly spherical shape with an average diameter of $30 \mathrm{~nm}$ compared with that of MOF-5 hollow nanospheres $135 \mathrm{~nm}$ (ref. 32)

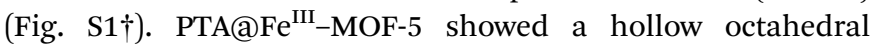
nanostructure as displayed in Fig. 1 without distinct change

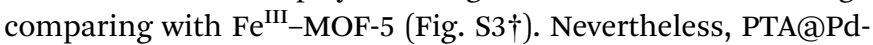
$\mathrm{Cu} @ \mathrm{Fe}^{\mathrm{III}}-\mathrm{MOF}-5$ indicated a polyhedron structure as shown in Fig. 3 attributing to the encapsulation of PTA in PdCu@Fe ${ }^{\mathrm{III}}$ MOF-5 (Fig. S4†). Energy-dispersive X-ray spectroscopy (EDS) analysis manifested that the elements $\mathrm{P}$ and $\mathrm{W}$ distributed throughout PTA@Fe ${ }^{\mathrm{III}}-\mathrm{MOF}-5$ and PTA@PdCu@Fe ${ }^{\mathrm{III}}-\mathrm{MOF}-5$ homogeneously as shown in Fig. 2 and 4 respectively. ICP-OES confirmed that the concentration of the element $\mathrm{Pd}, \mathrm{Cu}$ and $\mathrm{W}$ was $9.7 \mathrm{mM} \mathrm{L}^{-1}, 10.3 \mathrm{mM} \mathrm{L}^{-1}$ and $0.3 \mathrm{mM} \mathrm{L}^{-1}$ respectively.

XRD analysis of PTA@PdCu@Fe ${ }^{\mathrm{III}}-\mathrm{MOF}-5, \mathrm{PdCu} @ \mathrm{Fe}^{\mathrm{III}}$ MOF-5, PTA@Fe ${ }^{\mathrm{III}}-\mathrm{MOF}-5$ and PdCu dendrites were displayed in Fig. 5. XRD patterns of PTA@Fe ${ }^{\mathrm{III}}-\mathrm{MOF}-5, \mathrm{PdCu} @ \mathrm{Fe}^{\mathrm{III}}-\mathrm{MOF}-$ 5 and PTA@PdCu@Fe ${ }^{\mathrm{III}}-\mathrm{MOF}-5$ are almost identical to that of $\mathrm{Fe}^{\mathrm{III}}$-MOF-5 (Fig. S10 $\dagger$ ), respectively. This confirms that PTA should be encapsulated into $\mathrm{Fe}^{\mathrm{III}}$-MOF-5 without disturbing the MOF structure. Moreover, no patterns belonging to PTA crystals were observed suggesting that PTA is highly dispersed in the cage of $\mathrm{Fe}^{\mathrm{III}}-\mathrm{MOF}-5$ by small clusters. ${ }^{43}$ The peaks at $41.5^{\circ}$ and $48^{\circ}$ of PdCu dendrites coincided with the (111) and (200) peak of PdCu alloys (JCPDS 48-1551), ${ }^{\mathbf{4 2 , 4 4}}$ respectively. The element $\mathrm{P}, \mathrm{W}$, $\mathrm{Pd}$ and $\mathrm{Cu}$ were not detected in X-ray diffraction patterns of

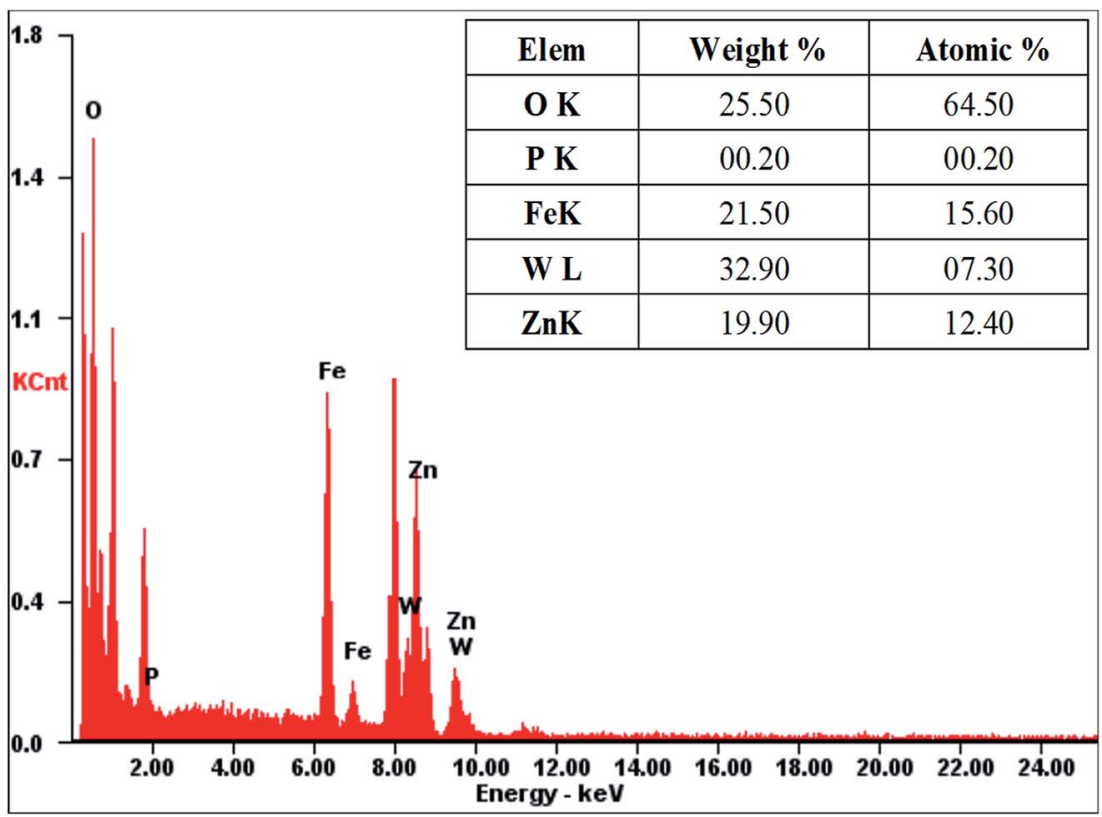

Fig. 2 EDS analysis of PTA@Fe ${ }^{\prime \prime \prime}-M O F-5$. 


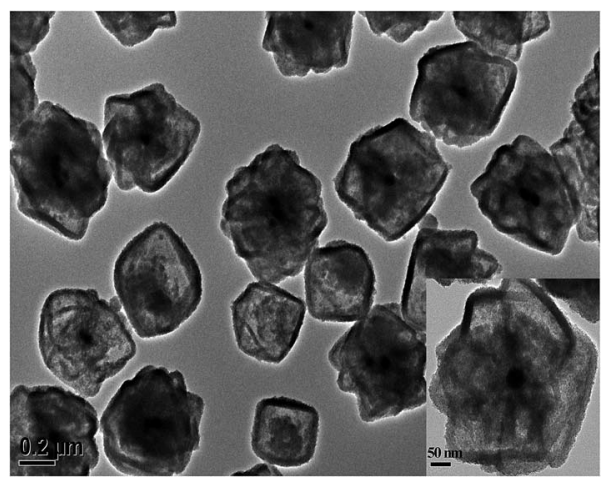

Fig. 3 TEM image of PTA@PdCu@Fe ${ }^{\prime \prime \prime}-M O F-5$.

PTA@Fe ${ }^{\mathrm{III}}-\mathrm{MOF}-5$, PTA@PdCu@Fe ${ }^{\mathrm{III}}-\mathrm{MOF}-5$ and PdCu@Fe ${ }^{\mathrm{III}}-$ MOF-5, indicating a good confinement effect of $\mathrm{Fe}^{\mathrm{III}}-\mathrm{MOF}-5$ hollow octahedral nanostructure for PdCu dendrites and PTA.

Acidic properties of PTA@PdCu@Fe ${ }^{\mathrm{III}}-\mathrm{MOF}-5$ and PdCu@$\mathrm{Fe}^{\mathrm{III}}$-MOF-5 characterized by $\mathrm{NH}_{3}$-TPD were shown in Fig. 6 . The distinct peak of PTA@PdCu@Fe ${ }^{\mathrm{III}}$-MOF-5 observed in the range of $150-250{ }^{\circ} \mathrm{C}$ corresponded to the weak acid sites. ${ }^{45,46}$ Compared to $\mathrm{PdCu} @ \mathrm{Fe}^{\mathrm{III}}$-MOF-5 the acidic property of PTA@PdCu@Fe ${ }^{\mathrm{III}}$-MOF-5 was considerably pronounced after the encapsulation of PTA.

TG and DTG analysis of the as-obtained catalysts in nitrogen were shown in Fig. 7. Weight losses below $150{ }^{\circ} \mathrm{C}$ are associated with the loss of hygroscopic water by dehydration. In the temperature range $200-300{ }^{\circ} \mathrm{C}$, the weight losses are attributed to the solvent liberation..$^{47}$ Above $400{ }^{\circ} \mathrm{C}$, weight losses can be attributed to the thermal decomposition of MOF structures. The TG and DTG analysis demonstrated that PTA@PdCu@Fe ${ }^{\mathrm{III}}$

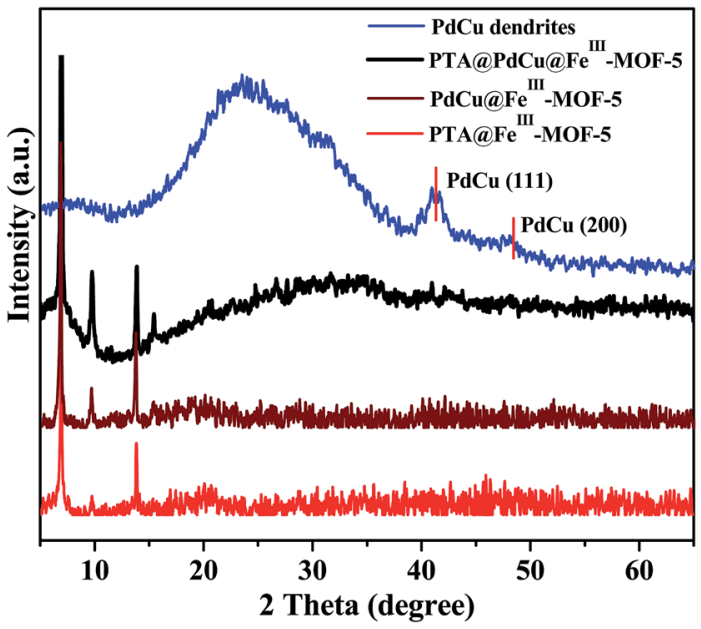

Fig. 5 XRD analysis of PTA@PdCu@Fe"II-MOF-5, PdCu@Fe ${ }^{\prime \prime \prime}-M O F-5$, PTA@Fe ${ }^{\text {III }}-$ MOF-5 and PdCu dendrites.

MOF-5 polyhedral nanostructures did not exhibit significant weight loss up to at least $350{ }^{\circ} \mathrm{C}$, and indicated a good thermal stability below $350{ }^{\circ} \mathrm{C}$ of both PTA@PdCu@Fe ${ }^{\mathrm{III}}-\mathrm{MOF}-5$ and PdCu@Fe ${ }^{\mathrm{III}}-\mathrm{MOF}-5$.

The permanent porosity of the as-obtained MOFs was confirmed by pore size distribution measurements and nitrogen adsorption-desorption isotherms as shown in Fig. 8 and 9 respectively. As shown in Fig. 9, both PdCu@Fe ${ }^{\mathrm{III}}-\mathrm{MOF}-5$ and PdCu@PTA@Fe ${ }^{\mathrm{III}}-\mathrm{MOF}-5$ exhibited a typical type IV sorption isotherm, indicating mesoporosity of the MOFs. ${ }^{48}$ Additionally, it was observed that nitrogen uptake displays an obvious increase at a relatively low pressure $\left(P / P_{0}<0.01\right)$, suggesting the presence of inherent micropores in these hollow

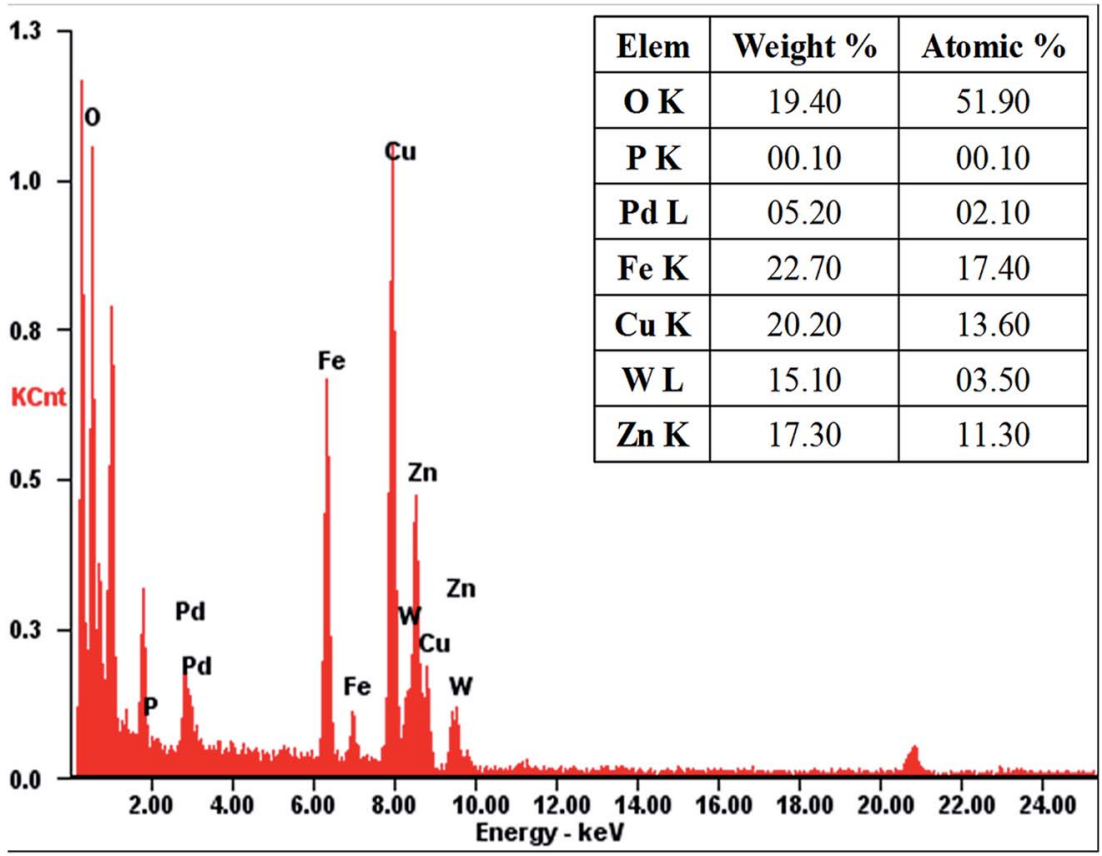

Fig. 4 EDS analysis of PTA@PdCu@Fe ${ }^{\text {III }-M O F-5 . ~}$ 


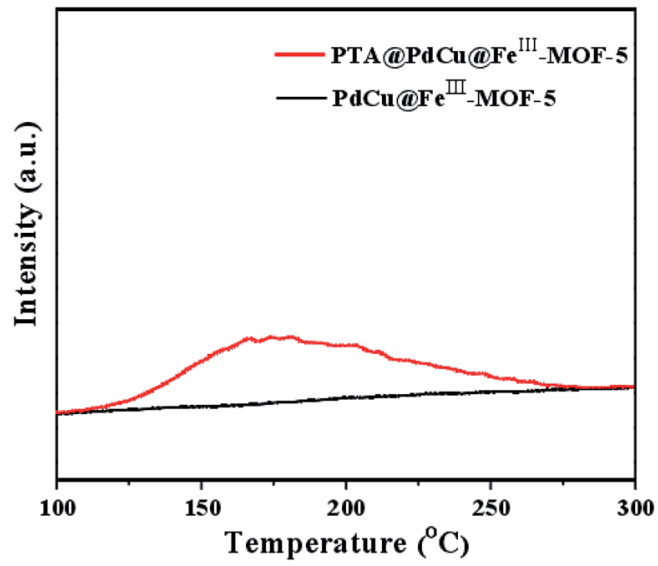

Fig. $6 \mathrm{NH}_{3}$-TPD analysis of PTA@PdCuaFe"II-MOF-5 and PdCuaFe $\mathrm{ell}^{\mathrm{II}}-\mathrm{MOF}-5$.

nanocages. ${ }^{32,49}$ The pore size distribution calculated from the $\mathrm{BJH}$ method using the desorption branch in Fig. 8 showed that the shells of PdCu@Fe ${ }^{\mathrm{III}}-\mathrm{MOF}-5$ and PTA@PdCu@Fe ${ }^{\mathrm{III}}-\mathrm{MOF}-5$
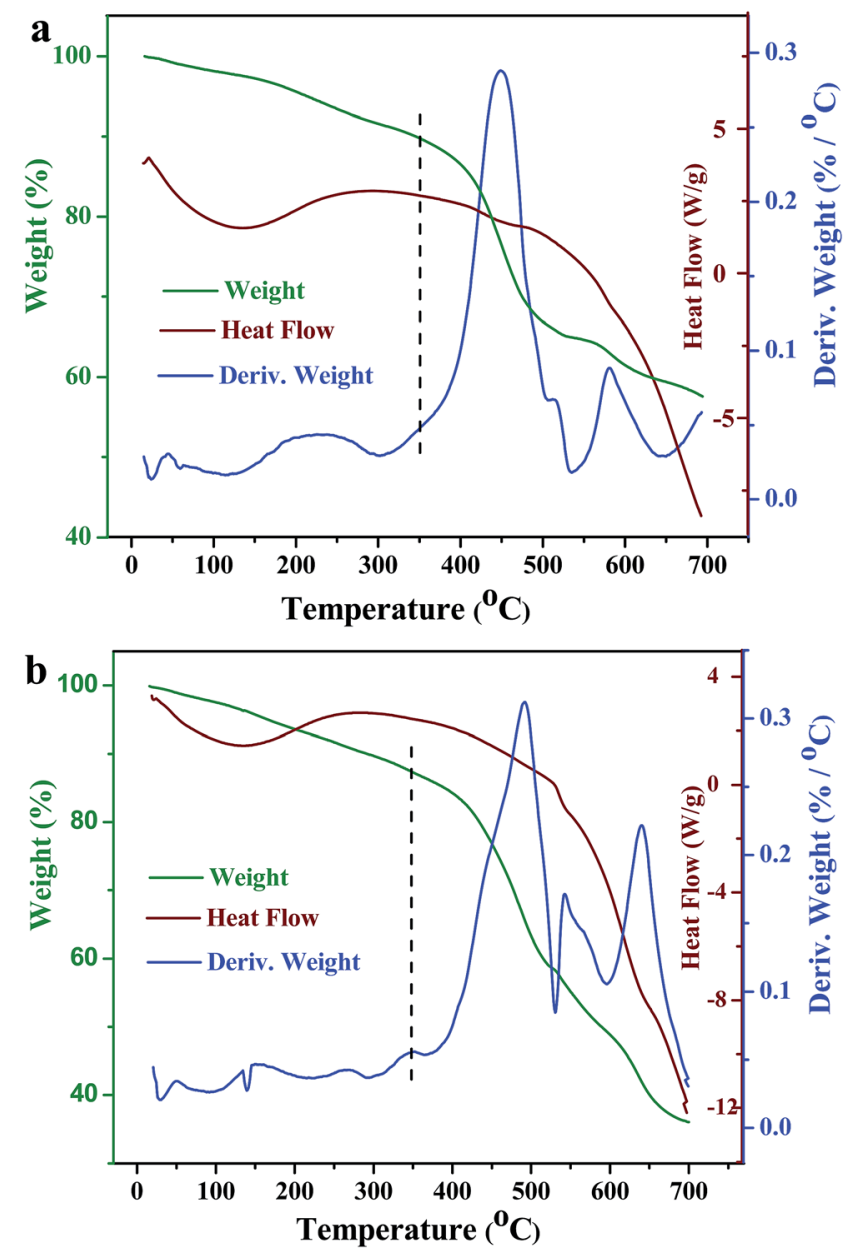

Fig. 7 Thermogravimetric (TG) analysis and derivative thermogravimetric (DTG) (rate of weight loss) of (a) the yolk-shell PTA@PdCu@Fe"II-MOF-5 and (b) PdCuaFe ${ }^{\text {III }}-$ MOF-5 in nitrogen. Heating rate $10{ }^{\circ} \mathrm{C} \mathrm{min}^{-1}$ from room temperature to $700{ }^{\circ} \mathrm{C}$.

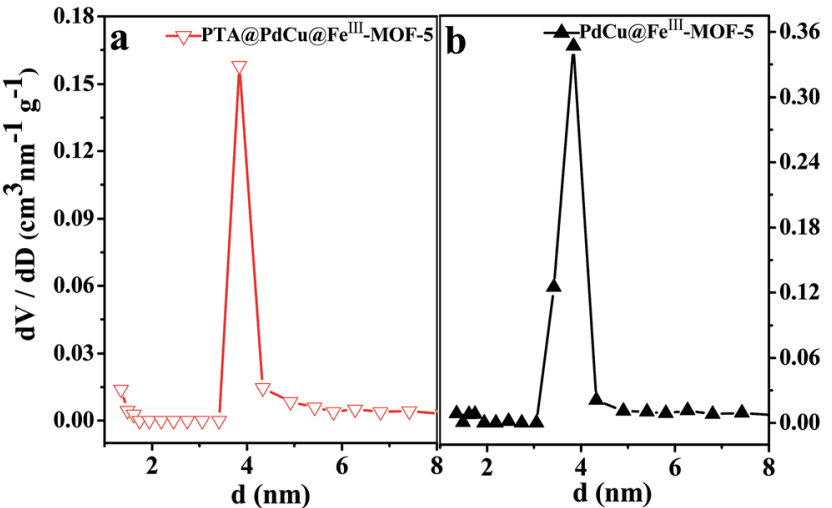

Fig. 8 Pore size distribution of PTA@PdCu@Fe"II-MOF-5 (a) and PdCuaFe III-MOF-5 (b) calculated from the BJH method using desorption branch.

mainly contain mesopores with diameters of $3.83 \mathrm{~nm}$ and $3.84 \mathrm{~nm}$ respectively. Due to the mesopores and micropores of the yolk-shell MOFs, PdCu@Fe ${ }^{\mathrm{III}}-\mathrm{MOF}-5$ and PTA@PdCu@Fe ${ }^{\mathrm{III}}$-MOF-5 shows a BET surface area of $240.3 \mathrm{~m}^{2} \mathrm{~g}^{-1}$ and $188.3 \mathrm{~m}^{2} \mathrm{~g}^{-1}$ respectively. The pore volumes of $\mathrm{PdCu} @ \mathrm{Fe}^{\mathrm{III}}$ MOF-5 and PTA@PdCu@Fe ${ }^{\mathrm{III}}-\mathrm{MOF}-5$ are 0.40 and $0.17 \mathrm{~cm}^{3} \mathrm{~g}^{-1}$, respectively. The encapsulation of PTA inside $\mathrm{PdCu@Fe} \mathrm{FII}^{\mathrm{II}}$ MOF-5 structure resulted in the decrease of pore volume and BET surface area of PTA@PdCu@Fe ${ }^{\mathrm{III}}-\mathrm{MOF}-5$. The voids between coordination polymer aggregated in the shells presumably formed the mesopores of the catalysts. ${ }^{50}$

As shown in Fig. 10, the FT-IR spectra of $\mathrm{Fe}^{\mathrm{III}}-\mathrm{MOF}-5$ and PdCu@Fe ${ }^{\mathrm{III}}$-MOF-5 hollow octahedral nanostructures verify the coordination of the carboxylate groups to metal ions, as evidenced by a red shift in the carboxylate stretching frequency to $1575 \mathrm{~cm}^{-1}$ from $1680 \mathrm{~cm}^{-1}$ for uncoordinated $\mathrm{H}_{2} \mathrm{BDC}$, which is consistent with the previous report. ${ }^{32,51}$ Meanwhile, the carboxylate stretching frequency of PTA@Fe ${ }^{\mathrm{III}}-\mathrm{MOF}-5$ and

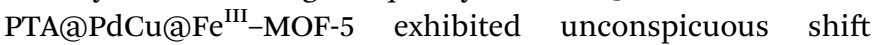
compared with Fe ${ }^{\mathrm{III}}-\mathrm{MOF}-5$. And the XPS spectra of PTA@Fe ${ }^{\mathrm{III}}$ MOF-5 hollow nanostructures (Fig. S11 $\dagger$ ) coincides with that of $\mathrm{Fe}^{\mathrm{III}}-\mathrm{MOF}-5 .^{32}$ Both TEM, FT-IR and XPS spectra analysis

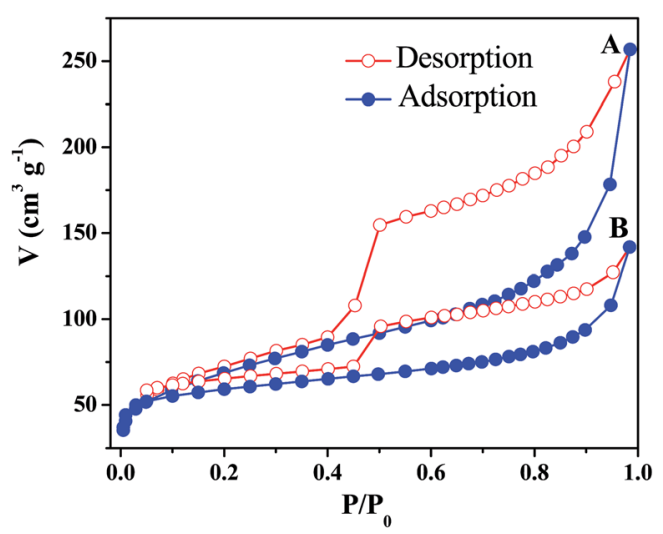

Fig. 9 Nitrogen adsorption-desorption isotherms of PdCu@Fe ${ }^{\text {III- }}$ MOF-5 (A) and PTA@PdCu@Fe"II-MOF-5 (B) measured at $77 \mathrm{~K}$. 


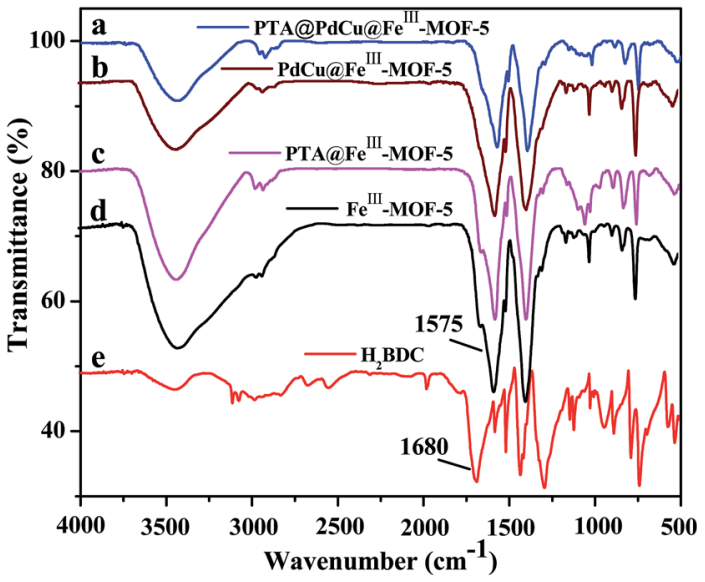

Fig. 10 FT-IR spectra of as-synthesized PTA@PdCu@Fe III-MOF-5 (a),

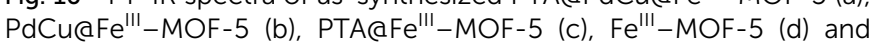
$\mathrm{H}_{2} \mathrm{BDC}(\mathrm{e})$.

confirmed that PTA were encapsulated inside the Fe ${ }^{\mathrm{III}}-\mathrm{MOF}-5$ structures and the structure of PTA@Fe ${ }^{\mathrm{III}}-\mathrm{MOF}-5$ maintained approximately the same with that of $\mathrm{Fe}^{\mathrm{III}}-\mathrm{MOF}-5$.

\subsection{Catalytic HDO of palmitic acid}

3.2.1 Effect of different as-prepared catalysts. The catalytic

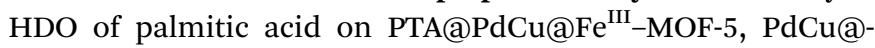
$\mathrm{Fe}^{\mathrm{III}}$-MOF-5, Pd NPs@Fe ${ }^{\mathrm{II}}-\mathrm{MOF}-5$, Au NPs@Fe ${ }^{\mathrm{III}}-\mathrm{MOF}-5, \mathrm{PdCu}$ dendrites, Pd NPs and Au NPs were indicated in Table 1. The SCF of $n$-hexane can be obtained at $240{ }^{\circ} \mathrm{C}$ and under the initial $2 \mathrm{MPa} \mathrm{H}_{2}$, hence $240{ }^{\circ} \mathrm{C}$ could be selected as the optimum reaction temperature. It can be concluded from Table 1 that the catalytic activity of PdCu@Fe ${ }^{\mathrm{III}}-\mathrm{MOF}-5, \mathrm{Pd} \mathrm{NPs} @ \mathrm{Fe}^{\mathrm{II}}-\mathrm{MOF}-5$ and $\mathrm{Au}$ NPs@Fe ${ }^{\mathrm{III}}-\mathrm{MOF}-5$ is remarkably superior to $\mathrm{Fe}^{\mathrm{III}}$ MOF-5, PTA@Fe ${ }^{\mathrm{III}}$-MOF-5, PdCu dendrites, Pd NPs and Au NPs respectively. It can be concluded from the entry 2, 3, 4 and entry 5 that metal active sites played a key role in catalytic HDO of palmitic acid. Moreover, the catalytic efficiency of the yolk-shell catalysts for the HDO reactions were increased remarkably attributing to the high hydrogen storage capacity of MOFs and the immobilization and dispersion effect of MOF for MNPs. Notably, the catalytic hydrogenation performance of bimetallic MOF system PdCu@Fe ${ }^{\mathrm{III}}-\mathrm{MOF}-5$ has an advantage over monometal MOF system i.e. Pd NPs@Fe ${ }^{\mathrm{II}}-\mathrm{MOF}-5$ and Au NPs@Fe ${ }^{\mathrm{II}}$ MOF-5. The products of catalytic HDO of palmitic acid mainly contained hexadecanal, hexadecanol and hexadecane. The catalytic HDO of palmitic acid over PdCu@Fe ${ }^{\mathrm{III}}-\mathrm{MOF}-5$ mainly produced hexadecanol. Compared to $\mathrm{PdCu} @ \mathrm{Fe}^{\mathrm{III}}-\mathrm{MOF}-5$, the products distribution manifested that the selectivity of hexadecane increased significantly on PTA@PdCu@Fe ${ }^{\mathrm{II}}-\mathrm{MOF}-5$ due to the enhancement of acidity of the catalyst. Compared to the catalyst exploited to the HDO of palmitic acid in the previous work, ${ }^{7,46}$ the yolk-shell PTA@PdCu@Fe ${ }^{\mathrm{III}}-\mathrm{MOF}-5$ exhibited a considerable advantage as can be seen in Table S1. $\dagger$

3.2.2 Effect of different reaction media. Table 2 showed the catalytic HDO of palmitic acid over PTA@PdCu@Fe ${ }^{\mathrm{III}}-\mathrm{MOF}-5$ and PdCu@Fe ${ }^{\mathrm{III}}-\mathrm{MOF}-5$ in supercritical $n$-hexane and conventional $n$-decane media respectively. As shown, the performance of the catalysts in SCF of $n$-hexane is superior to that in conventional $n$-decane media, the conversion of palmitic acid in SCF of $n$-hexane is about $15 \%$ higher than that in decane due to the good compatibility of the hydrogen and palmitic acid of SCF. The enhancement of acidity of PTA@PdCu@Fe ${ }^{\mathrm{III}}-\mathrm{MOF}-5$ contributed to the dehydration of hexadecanol to produce hexadecane. With the temperature increasing to $240{ }^{\circ} \mathrm{C}$ the final pressure of SCF system of $n$-hexane is approximately $0.3 \mathrm{MPa}$ lower than that of traditional $n$-decane system as a result of the good compatibility of the hydrogen of SCF of $n$-hexane and high hydrogen storage capacity of MOF materials.

\subsection{Catalytic HDO of palmitic acid over PTA@PdCu@Fe ${ }^{\mathrm{III}}$ MOF-5 in $n$-hexane}

3.3.1 Effect of reaction temperatures. Generally, reaction temperature directly affects the reaction rate and equilibrium. ${ }^{\mathbf{5 2}}$ As listed in Table 3, catalytic reaction over PTA@PdCu@Fe ${ }^{\mathrm{III}}$ MOF-5 at different reaction temperature showed that the conversion was relatively low at $180{ }^{\circ} \mathrm{C}$, but increased dramatically to $100 \%$ at $240{ }^{\circ} \mathrm{C}$ (the supercritical state of $n$-hexane). Analysis of product distribution revealed that when the temperature increased to $240{ }^{\circ} \mathrm{C}, \quad 93.7 \%$ selectivity of

Table 1 HDO of palmitic acid over different as-prepared catalysts ${ }^{a}$

\begin{tabular}{|c|c|c|c|c|c|}
\hline \multirow[b]{2}{*}{ Cat. } & \multirow[b]{2}{*}{ Conv. $\%$} & \multicolumn{4}{|l|}{ Selectivity $/ \%$} \\
\hline & & Hexadecanal & Hexadecanol & Hexadecane & Pentadecane \\
\hline PTA@PdCu@Fe & 100 & 0 & 5.3 & 90.6 & 4.1 \\
\hline $\mathrm{PdCu} @ \mathrm{Fe}^{\mathrm{III}}-\mathrm{MOF}-5$ & 100 & 0.2 & 74 & 24.8 & 1 \\
\hline Pd NPs@Fe ${ }^{\text {III-MOF-5 }}$ & 82.1 & 5.2 & 76.2 & 18.1 & 0.5 \\
\hline Au NPs@Fe ${ }^{\text {III }-M O F-5 ~}$ & 70.5 & 9.8 & 71.1 & 19.1 & 0 \\
\hline $\mathrm{Fe}^{\mathrm{III}}-\mathrm{MOF}-5$ & 3.6 & 19.1 & 80.9 & 0 & 0 \\
\hline PTA@Fe ${ }^{\mathrm{III}}-\mathrm{MOF}-5$ & 3.9 & 18.6 & 81.4 & 0 & 0 \\
\hline PdCu dendrites & 25.7 & 20.1 & 60.3 & 19.6 & 0 \\
\hline Pd NPs & 13.2 & 25.3 & 65 & 9.7 & 0 \\
\hline Au NPs & 10.5 & 29.2 & 64.3 & 6.5 & 0 \\
\hline
\end{tabular}

${ }^{a}$ Reaction conditions: solvent $30 \mathrm{~mL} n$-hexane, reactant palmitic acid $0.3 \mathrm{~g}$, temperature $240{ }^{\circ} \mathrm{C}$, catalyst $0.1 \mathrm{~g}$, initial $\mathrm{H}_{2}$ pressure $2 \mathrm{MPa}$, reaction time $2 \mathrm{~h}$. 
Table 2 Catalytic HDO of palmitic acid in SCF of $n$-hexane and conventional $n$-decane media ${ }^{a}$

\begin{tabular}{|c|c|c|c|c|c|c|}
\hline Media & Cat. & Conv./\% & \multicolumn{4}{|l|}{ Selectivity $/ \%$} \\
\hline & PdCu@Fe ${ }^{\mathrm{III}}-\mathrm{MOF}-5$ & 100 & 0.2 & 74 & 24.8 & 1 \\
\hline$n$-Decane & PTA@PdCu@Fe & 85.1 & 0.6 & 5.9 & 90.4 & 3.1 \\
\hline & PdCu@Fe ${ }^{\text {III }}-M O F-5$ & 84.6 & 0.1 & 74.9 & 24.2 & 0.8 \\
\hline
\end{tabular}

${ }^{a}$ Reaction conditions: solvent $n$-hexane $30 \mathrm{~mL}$, reactant palmitic acid $0.3 \mathrm{~g}$, temperature $240{ }^{\circ} \mathrm{C}$, catalyst $0.1 \mathrm{~g}$, initial $\mathrm{H}_{2}$ pressure $2 \mathrm{MPa}$, reaction time $2 \mathrm{~h}$.

Table 3 Catalytic HDO of palmitic acid over PTA@PdCu@Fe ${ }^{\prime \prime \prime}-$ MOF-5 and PdCu@Fe ${ }^{\text {III }}-$ MOF-5 in $n$-hexane ${ }^{a}$

\begin{tabular}{|c|c|c|c|c|c|c|}
\hline Cat. & $T /{ }^{\circ} \mathrm{C}$ & Conv./\% & Hexadecanal & Hexadecanol & Hexadecane & Pentadecane \\
\hline & 200 & 86.5 & 10.3 & 63.2 & 31.4 & 1.1 \\
\hline & 220 & 99.8 & 0 & 31.1 & 66.6 & 2.3 \\
\hline & 240 & 99.9 & 0 & 2.3 & 93.6 & 4.1 \\
\hline & 220 & 96.5 & 15.3 & 70 & 18.5 & 0.2 \\
\hline & 240 & 99.8 & 0 & 74 & 25 & 1 \\
\hline & 260 & 100 & 0 & 65.3 & 33.2 & 1.5 \\
\hline
\end{tabular}

${ }^{a}$ Reaction conditions: solvent $30 \mathrm{~mL} n$-hexane, reactant palmitic acid $0.3 \mathrm{~g}$, catalyst $0.1 \mathrm{~g}$, initial $\mathrm{H}_{2}$ pressure $2 \mathrm{MPa}$, reaction time $2 \mathrm{~h}$.

$n$-hexadecane was achieved. Further elevating the temperature to $260{ }^{\circ} \mathrm{C}$, the selectivity of hexadecane almost constant. Large amounts of hexadecanol could be obtained when conducting the reaction below $200{ }^{\circ} \mathrm{C}$ indicating that the dehydration of the hexadecanol has the highest temperature barrier in the whole reaction. The selectivity of the decarbonylation product pentadecane increased with a very small extent from $0 \%$ at $180{ }^{\circ} \mathrm{C}$ to $5.9 \%$ at $260{ }^{\circ} \mathrm{C}$. And the hydrocracking products were not detected during the reaction. Indicating that HDO is the main

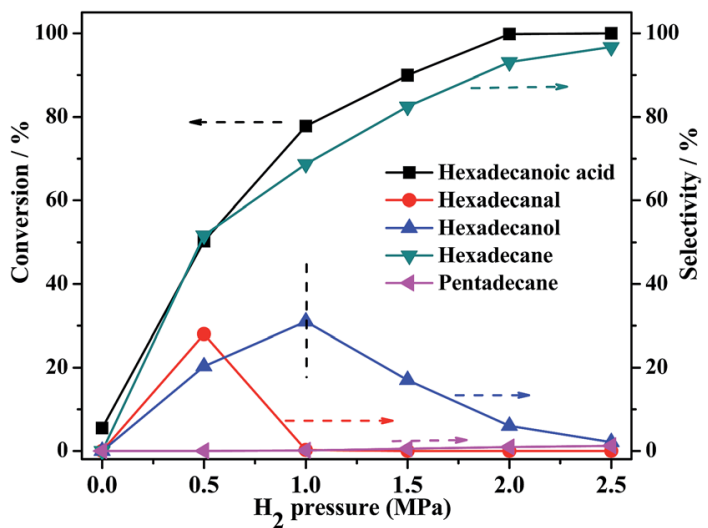

Fig. 11 Effect of $\mathrm{H}_{2}$ pressure on palmitic acid conversion and selectivity to hydrogenation products over PTA@PdCu@Fe ${ }^{\text {III-MOF-5. }}$ Reaction conditions: solvent $n$-hexane $30 \mathrm{~mL}$, palmitic acid $0.3 \mathrm{~g}$, catalyst $0.1 \mathrm{~g}$, reaction temperature $240{ }^{\circ} \mathrm{C}$, reaction time $2 \mathrm{~h}$. pathway. PdCu@Fe ${ }^{\mathrm{III}}$-MOF-5 were also investigated as the catalyst as displayed in Table 3 , the conversion increased from $78.1 \%$ at $200{ }^{\circ} \mathrm{C}$ to $99.8 \%$ at $240{ }^{\circ} \mathrm{C}$, the selectivity of hexadecanol increased from $67.2 \%$ at $200{ }^{\circ} \mathrm{C}$ to $74 \%$ at $240{ }^{\circ} \mathrm{C}$, then slightly decreased to $65.3 \%$ at $260{ }^{\circ} \mathrm{C}$. Whereas the selectivity of hexadecane was relatively low that increased from $11.6 \%$ at $200{ }^{\circ} \mathrm{C}$ to $33.2 \%$ at $260{ }^{\circ} \mathrm{C}$ as a result of the inacidity of

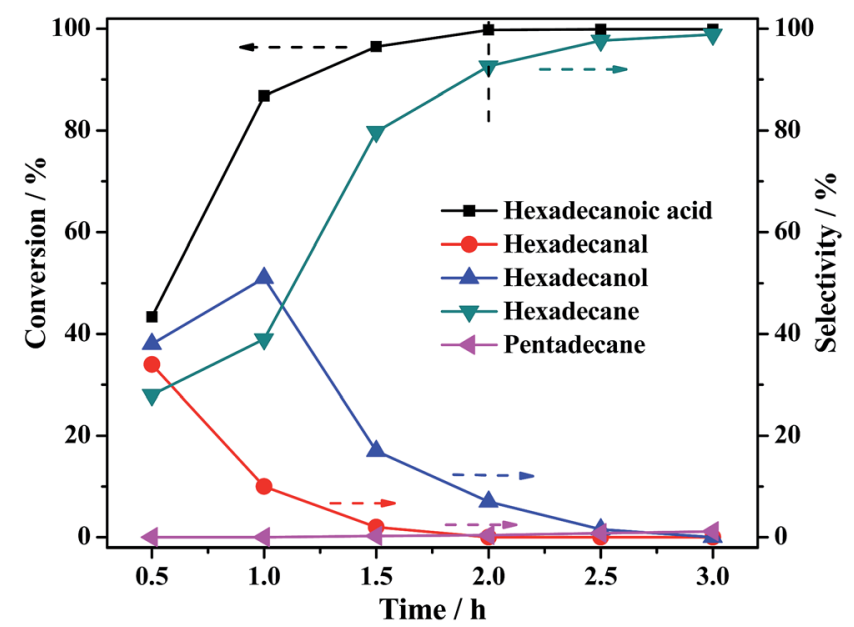

Fig. 12 Effect of reaction time on palmitic acid conversion and the selectivity of hydrogenation products over PTA@PdCu@Fe ${ }^{\text {III }}-$ MOF-5. Reaction conditions: solvent $n$-hexane $30 \mathrm{~mL}$, palmitic acid $0.3 \mathrm{~g}$, catalyst $0.1 \mathrm{~g}$, initial $\mathrm{H}_{2}$ pressure $2 \mathrm{MPa}$, reaction temperature $240{ }^{\circ} \mathrm{C}$. 


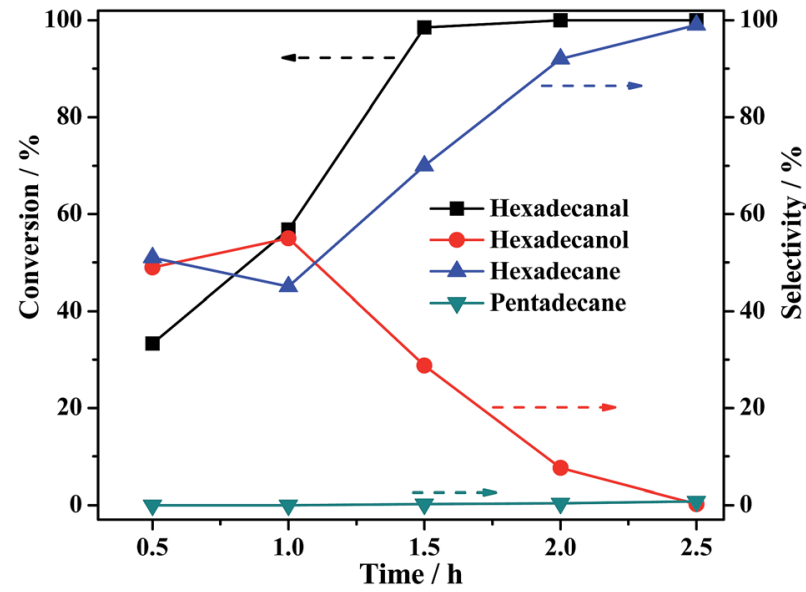

Fig. 13 Effect of reaction time on hexadecanal conversion and the selectivity of hydrogenation products over PTACPdCuaFe III-MOF-5. Reaction conditions: solvent $n$-hexane $30 \mathrm{~mL}$, palmitic acid $0.3 \mathrm{~g}$, catalyst $0.1 \mathrm{~g}$, initial $\mathrm{H}_{2}$ pressure $2 \mathrm{MPa}$, reaction temperature $240{ }^{\circ} \mathrm{C}$.

PdCu@Fe ${ }^{\text {III }}-\mathrm{MOF}-5$. The encapsulation of PTA inside the yolkshell composites enhanced the acidity of PTA@PdCu@Fe ${ }^{\mathrm{III}}$ MOF-5, thus, contributing to the dehydration of hexadecanol. ${ }^{53}$ The different acidity of PTA@PdCu@Fe ${ }^{\mathrm{II}}-\mathrm{MOF}-5$ and PdCu@$\mathrm{Fe}^{\mathrm{III}}$-MOF-5 elucidated the different products distribution of HDO of palmitic acid.

3.3.2 Effect of hydrogen pressure. Initial hydrogen pressure was an important parameter that affected the HDO rate of palmitic acid. The pressure variation experiments were investigated using PTA@PdCu@Fe ${ }^{\mathrm{III}}-\mathrm{MOF}-5$ as a catalyst under $240{ }^{\circ} \mathrm{C}$ as shown in Fig. 11. As expected, with the initial $\mathrm{H}_{2}$ pressure increased from 0 to $2.5 \mathrm{MPa}$ the conversion of palmitic acid increased from $5.5 \%$ to $100 \%$ and the selectivity of hexadecane increased from 0 to $96.7 \%$. And the selectivity of hexadecanal and hexadecanol increased first from 0 to $28 \%$ and $31 \%$ then decreased to 0 and $2.1 \%$ respectively. While the selectivity of the decarbonylation product $n$-pentadecane was very low (from 0 at $0 \mathrm{MPa}$ to $1.2 \%$ at $2.5 \mathrm{MPa}$ ). Hydrogen participated in converting hexadecanal into hexadecanol, the branching step in the reaction. Increasing the amount of $\mathrm{H}_{2}$ facilitates the equilibrium toward hexadecanol accelerating the pathway to $n$-hexadecane. Confirming that the main route is hydrogenation-dehydration-hydrogenation reactions to generate hexadecane without carbon loss. The result indicated that higher hydrogen pressure facilitates the HDO pathway over PTA@PdCu@Fe ${ }^{\mathrm{II}}$-MOF-5 coinciding with previous reports. ${ }^{54-56}$

3.3.3 Effect of reaction time. For the study of reaction mechanism, the effect of reaction time on the catalytic HDO of palmitic acid was also studied as depicted in Fig. 12. The conversion of palmitic acid reached almost $100 \%$ after reacting for 2 h. Fig. 12 reveals that the selectivity of hexadecanol gradually increased from $38 \%$ at $0.5 \mathrm{~h}$ to $51 \%$ at $1 \mathrm{~h}$, and then gradually decreased to $0 \%$ at $3 \mathrm{~h}$, along with that of hexadecanal decreased consecutively from $34 \%$ at $0.5 \mathrm{~h}$ to $0 \%$ at $3 \mathrm{~h}$. Whereas that of hexadecane increased continuously to $99.8 \%$ at $3 \mathrm{~h}$. And the selectivity of pentadecane remained very low during the reaction. It is observed that palmitic acid exhausted earlier than hexadecanol. A fast hydrogenation reduction of palmitic acid to hexadecanal proceeded firstly, immediately following by the hydrogenation of hexadecanal to generate hexadecanol. Then the main product hexadecane was produced by slow dehydration of hexadecanol. The reduction rate of hexadecanal to hexadecanol was confirmed to be faster than the production rate of hexadecanal over PTA@PdCu@Fe ${ }^{\mathrm{III}}-\mathrm{MOF}-5$.

\section{Mechanism study}

Hexadecanal was placed to the reaction conditions to confirm that hexadecanol was generated from the hydrogenation of hexadecanal. As shown in Fig. 13, the selectivity of hexadecanol gradually increased from $49 \%$ at $0.5 \mathrm{~h}$ to $55 \%$ at $1 \mathrm{~h}$ and then decreased to $2.2 \%$ at $3 \mathrm{~h}$, meanwhile the selectivity of the HDO product hexadecane increased from $51 \%$ to $99 \%$, indicating that HDO was the main pathway for the conversion of hexadecanal over PTA@PdCu@Fe ${ }^{\mathrm{II}}$-MOF-5. Conversely, the selectivity of the decarbonylation product (pentadecane) was less than $1 \%$. This suggests that hexadecanol obtained from hexadecanal is through a hydrogenation mechanism as displays in Scheme 1. The (de)hydrogenation between hexadecanol and hexadecanal is reversible. Hexadecane was generated from the hydrogenation of hexadecene that came from the dehydration of hexadecanol.

\section{Conclusions}

A series of well-defined MOF hollow nanostructures encapsulated with PTA (PTA@MOF-5, PTA@Fe ${ }^{\mathrm{III}}-\mathrm{MOF}-5$, PTA@Pd$\left.\mathrm{Cu} @ \mathrm{Fe}^{\mathrm{III}}-\mathrm{MOF}-5\right)$ were successfully synthesized by a facile solvothermal approach. It is demonstrated that the

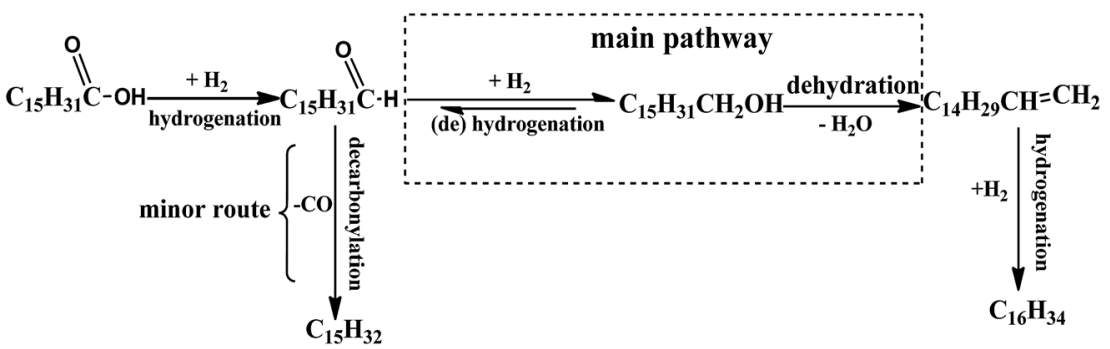

Scheme 1 Proposed hydrogenation process for palmitic acid over PTA@PdCu@Fe ${ }^{\prime \prime \prime}-M O F-5$. 
performance of the catalysts in SCF of $n$-hexane is superior to that in the conventional media. Selective hydrogenation results of palmitic acid indicate that the yolk-shell PTA@PdCu@Fe ${ }^{\mathrm{III}}$ MOF-5 nanostructures is in favor of achieving excellent catalytic performance as compared to the pure noble-metal nanocrystals and other catalysts used in the previous work. The acidic property of PTA@PdCu@Fe ${ }^{\mathrm{III}}-\mathrm{MOF}-5$ was greatly pronounced compared to $\mathrm{PdCu} @ \mathrm{Fe}^{\mathrm{III}}-\mathrm{MOF}-5$, resulting in the high selectivity of hexadecane. The high performance in the catalytic HDO of palmitic acid is associated with the synergistic effect between yolk-shell PTA@PdCu@Fe ${ }^{\mathrm{III}}$-MOF-5 nanostructures and SCF. The present work demonstrated a new green catalytic process towards the hydrogenation upgrading of algal-based bio-oil and a great potential in catalyzing organic reactions.

\section{Acknowledgements}

The authors are grateful for the financial supports from the National Natural Science Foundation of China (No. 21576155, 21376136 and No. 21376140), Research Project of Guangdong Provincial Department of Science and Technology Department (No. 2015B020215004).

\section{References}

1 P. Collet, L. Lardon, A. Hélias, S. Bricout, I. Lombaert-Valot, B. Perrier, O. Lépine, J.-P. Steyer and O. Bernard, Renewable Energy, 2014, 71, 525-533.

2 Z. Shuping, W. Yulong, Y. Mingde, I. Kaleem, L. Chun and J. Tong, Energy, 2010, 35, 5406-5411.

3 E. P. Bennion, D. M. Ginosar, J. Moses, F. Agblevor and J. C. Quinn, Appl. Energy, 2015, 154, 1062-1071.

4 Y. Chen, Y. Wu, D. Hua, C. Li, M. P. Harold, J. Wang and M. Yang, RSC Adv., 2015, 5, 18673-18701.

5 P. Duan and P. E. Savage, Bioresour. Technol., 2011, 102, 1899-1906.

6 S. Zou, Y. Wu, M. Yang, C. Li and J. Tong, Energy Environ. Sci., 2010, 3, 1073-1078.

7 R. Ding, Y. Wu, Y. Chen, J. Liang, J. Liu and M. Yang, Chem. Eng. Sci., 2015, 135, 517-525.

8 A. Baiker, Chem. Rev., 1999, 99, 453-474.

9 B. Subramaniam, Appl. Catal., A, 2001, 212, 199-213.

10 P. Pollet, E. A. Davey, E. E. Ureña-Benavides, C. A. Eckert and C. L. Liotta, Green Chem., 2014, 16, 1034.

11 M. Tsang, G. Philippot, C. Aymonier and G. Sonnemann, Green Chem., 2016, 18, 4924-4933.

12 Z. Sun, G. Bottari and K. Barta, Green Chem., 2015, 17, 51725181.

13 D. Verma, R. Insyani, Y.-W. Suh, S. M. Kim, S. K. Kim and J. Kim, Green Chem., 2017, 19, 1969-1982.

14 W. Li, C. Pan, Q. Zhang, Z. Liu, J. Peng, P. Chen, H. Lou and X. Zheng, Bioresour. Technol., 2011, 102, 4884-4889.

15 Q. Dang, Z. Luo, J. Zhang, J. Wang, W. Chen and Y. Yang, Fuel, 2013, 103, 683-692.

16 H. Wan, R. V. Chaudhari and B. Subramaniam, Top. Catal., 2012, 55, 129-139.
17 J. W. Ford, R. V. Chaudhari and B. Subramaniam, Ind. Eng. Chem. Res., 2010, 49, 10852-10858.

18 W. Shi, Y. Gao, S. Song and Y. Zhao, Ind. Eng. Chem. Res., 2014, 53, 11557-11565.

19 B. Panella, M. Hirscher, H. Pütter and U. Müller, Adv. Funct. Mater., 2006, 16, 520-524.

20 A. G. Wong-Foy, A. J. Matzger and O. M. Yaghi, J. Am. Chem. Soc., 2006, 128, 3494-3495.

21 H. Liu, L. Chang, C. Bai, L. Chen, R. Luque and Y. Li, Angew. Chem., Int. Ed., 2016, 55, 5019-5023.

22 Z. Hu, B. J. Deibert and J. Li, Chem. Soc. Rev., 2014, 43, 58155840.

23 H. Furukawa, K. E. Cordova, M. O'Keeffe and O. M. Yaghi, Science, 2013, 341, 1230444.

24 Z. Zhang, Y. Chen, S. He, J. Zhang, X. Xu, Y. Yang, F. Nosheen, F. Saleem, W. He and X. Wang, Angew. Chem., Int. Ed., 2014, 126, 12725-12729.

25 J. Yang, F. Zhang, H. Lu, X. Hong, H. Jiang, Y. Wu and Y. Li, Angew. Chem., Int. Ed., 2015, 54, 10889-10893.

26 K. Schlichte, T. Kratzke and S. Kaskel, Microporous Mesoporous Mater., 2004, 73, 81-88.

27 S. Hermes, M. K. Schroter, R. Schmid, L. Khodeir, M. Muhler, A. Tissler, R. W. Fischer and R. A. Fischer, Angew. Chem., Int. Ed., 2005, 44, 6237-6241.

28 M. Zhao, K. Yuan, Y. Wang, G. Li, J. Guo, L. Gu, W. Hu, H. Zhao and Z. Tang, Nature, 2016, 539, 76-80.

29 D.-L. Chen, S. Wu, P. Yang, S. He, L. Dou and F.-F. Wang, J. Phys. Chem. C, 2017, 121, 8857-8863.

30 F. Zhang, S. Zheng, Q. Xiao, Y. Zhong, W. Zhu, A. Lin and M. Samy El-Shall, Green Chem., 2016, 18, 2900-2908.

31 X. Xu, Z. Zhang and X. Wang, Adv. Mater., 2015, 27, 53655371.

32 Z. Zhang, Y. Chen, X. Xu, J. Zhang, G. Xiang, W. He and X. Wang, Angew. Chem., Int. Ed., 2014, 53, 429-433.

33 R. Canioni, C. Roch-Marchal, F. Sécheresse, P. Horcajada, C. Serre, M. Hardi-Dan, G. Férey, J.-M. Greneche, F. Lefebvre and J.-S. Chang, J. Mater. Chem., 2011, 21, 1226-1233.

34 R. Yu, X.-F. Kuang, X.-Y. Wu, C.-Z. Lu and J. P. Donahue, Coord. Chem. Rev., 2009, 253, 2872-2890.

35 L. Bromberg, Y. Diao, H. Wu, S. A. Speakman and T. A. Hatton, Chem. Mater., 2012, 24, 1664-1675.

36 R. Palkovits, K. Tajvidi, A. M. Ruppert and J. Procelewska, Chem. Commun., 2011, 47, 576-578.

37 Y. Zhang, V. Degirmenci, C. Li and E. J. Hensen, ChemSusChem, 2011, 4, 59-64.

38 D. Farrusseng, Metal-organic frameworks: applications from catalysis to gas storage, John Wiley \& Sons, 2011.

39 B. Chen, N. W. Ockwig, A. R. Millward, D. S. Contreras and O. M. Yaghi, Angew. Chem., Int. Ed., 2005, 117, 4823-4827.

40 B. Kesanli, Y. Cui, M. R. Smith, E. W. Bittner, B. C. Bockrath and W. Lin, Angew. Chem., Int. Ed., 2005, 44, 72-75.

41 A. G. Wong-Foy, A. J. Matzger and O. M. Yaghi, J. Am. Chem. Soc., 2006, 128, 3494-3495.

42 B. Xu, Z. Zhang and X. Wang, Nanoscale, 2013, 5, 4495-4505.

43 J. Juan-Alcañiz, E. V. Ramos-Fernandez, U. Lafont, J. Gascon and F. Kapteijn, J. Catal., 2010, 269, 229-241. 
44 L. Wang, J.-J. Zhai, K. Jiang, J.-Q. Wang and W.-B. Cai, Int. J. Hydrogen Energy, 2015, 40, 1726-1734.

45 L. Vilcocq, R. Koerin, A. Cabiac, C. Especel, S. Lacombe and D. Duprez, Appl. Catal., B, 2014, 148-149, 499-508.

46 Y. Duan, Y. Wu, Q. Zhang, R. Ding, Y. Chen, J. Liu and M. Yang, J. Mol. Catal. A: Chem., 2015, 398, 72-78.

47 S.-L. Zhong, R. Xu, L.-F. Zhang, W.-G. Qu, G.-Q. Gao, X.-L. Wu and A.-W. Xu, J. Mater. Chem., 2011, 21, 16574-16580.

48 D. P. Serrano, J. Aguado, J. M. Escola, J. M. Rodríguez and Á. Peral, Chem. Mater., 2006, 18, 2462-2464.

49 Y. Y. Karabach, M. F. C. Guedes da Silva, M. N. Kopylovich, B. Gil-Hernandez, J. Sanchiz, A. M. Kirillov and A. J. Pombeiro, Inorg. Chem., 2010, 49, 11096-11105.
50 S.-L. Zhong, R. Xu, L.-F. Zhang, W.-G. Qu, G.-Q. Gao, X.-L. Wu and A.-W. Xu, J. Mater. Chem., 2011, 21, 16574-16580.

51 W. Cho, H. J. Lee and M. Oh, J. Am. Chem. Soc., 2008, 130, 16943-16946.

52 X. Fang, Z. Yin, H. Wang, J. Li, X. Liang, J. Kang and B. He, J. Catal., 2015, 329, 187-194.

53 Y. Zhang, V. Degirmenci, C. Li and E. J. Hensen, ChemSusChem, 2011, 4, 59-64.

54 K. Kandel, J. W. Anderegg, N. C. Nelson, U. Chaudhary and I. I. Slowing, J. Catal., 2014, 314, 142-148.

55 L. Boda, G. Onyestyák, H. Solt, F. Lónyi, J. Valyon and A. Thernesz, Appl. Catal., A, 2010, 374, 158-169.

56 R. Ding, Y. Wu, Y. Chen, H. Chen, J. Wang, Y. Shi and M. Yang, Catal. Sci. Technol., 2016, 6, 2065-2076. 